\title{
Stability Analysis of Bulk Viscous Cosmology
}

\author{
M. Sharif ${ }^{1 \star}$ and Saadia Mumtaz ${ }^{1 \star \star}$ \\ ${ }^{1}$ Department of Mathematics, University of the Punjab, \\ Quaid-e-Azam Campus, Lahore-54590, Pakistan.
}

\begin{abstract}
In this paper, we study phase space analysis of FRW universe model by taking a power-law model for bulk viscosity coefficient. An autonomous system of equations is developed by defining normalized dimensionless variables. We find corresponding critical points for different values of the parameters to investigate stability of the system. It is found that the presence of power-law model of bulk viscosity appears as an effective ingredient to enhance the stability of the respective universe model.
\end{abstract}

\section{Introduction}

Many astronomical observations (type Ia supernova and cosmic microwave background radiation) have suggested that our universe is undergoing an accelerated expansion [1]. An unusual antigravitational force, known as dark energy (DE), is considered to be responsible for the current cosmic expansion. There are various alternative candidates for DE to study its mysterious characteristics among which the cosmological constant $(\Lambda)$ is the simplest one. It has also been suggested that bulk viscous fluids play an important role for accelerated cosmic expansion [2]. Bulk viscosity is one of the favorable dissipative contributions in homogeneous universe scenarios.

A phase space analysis is useful to study different patterns of evolution by transforming the system of equations to an autonomous one. Guo et al. [3] studied phase space analysis for FRW universe model filled with barotropic fluid as well as phantom scalar field and found that phantom dominated solution is a stable late-time attractor. Yang and Gao [4] explored this analysis for k-essence cosmology and obtained that stability of the critical points play a substantial role for the cosmic evolution. Recently, we have discussed the stability of accelerated expansion via phase space analysis for both isotropic [5] as well as anisotropic [6] universe models.

This work is devoted to study stability of FRW universe model by taking bulk viscous fluid through phase space analysis. The plan of the paper is as follows. In Sect. 2, we provide some basic equations and a power-law of bulk viscosity parameter. We study phase space analysis for both matter as well as radiation dominated universe model. We conclude our results in the last section.

\section{General Equations and Phase Space Analysis}

We consider homogeneous and isotropic universe model defined by the line element

$$
d s^{2}=-d t^{2}+a^{2}(t)\left(d r^{2}+r^{2} d \theta^{2}+r^{2} \sin ^{2} \theta d \phi^{2}\right),
$$

${ }^{\star}$ e-mail: msharif.math@pu.edu.pk

${ }^{\star}$ e-mail: sadiamumtaz17@gmail.com 
where $a(t)$ is the scale factor. The matter distribution for the cosmic fluid is given by

$$
T_{\mu \nu}=(\rho+\tilde{p}) u_{\mu} u_{v}+\tilde{p} g_{\mu \nu},
$$

where $\rho$ and $\tilde{p}$ correspond to the energy density and total pressure, respectively. The effective bulk viscous pressure is defined by the Eckart formalism as [7]

$$
\tilde{p}=p-3 \eta H,
$$

where $\eta$ represents bulk viscosity coefficient. The bulk viscosity gives dissipative effects to the cosmic fluid. The Friedmann equations describing the evolution of the respective universe model are

$$
\begin{gathered}
3 H^{2}=\rho, \\
2 \dot{H}+3 H^{2}=-\tilde{p},
\end{gathered}
$$

where $H=\frac{\dot{a}}{a}$ is the Hubble parameter and dot means derivative with respect to time. The conservation of energy-momentum tensor yields

$$
\dot{\rho}+3(\rho+\tilde{p}) H=0 .
$$

We consider a power-law model for bulk viscosity [8]

$$
\eta=\xi H^{\delta},
$$

where $\xi$ and $\delta$ are free parameters.

\subsection{Bulk Viscous Matter Dominated Universe}

Her we study stability of FRW model through phase space analysis dominated by matter (neglecting radiation). We define the dimensionless bulk viscous parameters as

$$
\tilde{\eta}=\frac{3 \eta}{H_{0}^{\delta}}
$$

which takes the form

$$
\tilde{\eta}=3 \xi\left(\frac{H}{H_{0}}\right)^{\delta},
$$

where $H_{0}$ defines the current value of Hubble parameter. The constraint and Raychaudhuri equations turn out to be

$$
\begin{gathered}
3 H^{2}=\rho_{m}, \\
H H_{0} \tilde{\eta}=2 \dot{H}+3 H^{2},
\end{gathered}
$$

where $\rho_{m}$ corresponds to the matter density. The conservation equation for bulk viscous dust becomes

$$
\dot{\rho}_{m}+3\left(\rho_{m}-H H_{0} \tilde{\eta}\right) H=0 .
$$

The deceleration and EoS parameter are defined by

$$
q=-1-\frac{\dot{H}}{H^{2}}, \quad \omega=-1-\frac{2 \dot{H}}{3 H^{2}},
$$


which, through Eqs.(9) and (11), yield

$$
\begin{gathered}
q=\frac{1}{2}\left[1-3 \xi\left(\frac{H}{H_{0}}\right)^{\delta-1}\right], \\
\omega=-\xi\left(\frac{H}{H_{0}}\right)^{\delta-1} .
\end{gathered}
$$

To find analytical solution of the evolution equations, we define normalized dimensionless variables as

$$
x=\Psi_{m}=\frac{\rho_{m}}{3 H^{2}}, \quad z=\frac{1}{1+\frac{H_{0}}{H}},
$$

which can reduce this system to an autonomous one. The system of Eqs.(11) and (12) in terms of normalized variables become

$$
\begin{gathered}
x^{\prime}=3 \xi(1-x)\left(\frac{1-z}{z}\right)^{1-\delta}, \\
z^{\prime}=\frac{3(1-z)}{2}\left[\xi z^{\delta}(1-z)^{1-\delta}-z\right] .
\end{gathered}
$$

We define a new variable $\tau$ for time such that $\frac{d t}{d \tau}=\frac{1}{H^{2}}$. The corresponding derivative will be represented by prime. In order to determine the critical points $\left(x_{c}, z_{c}\right)$, we need to solve the respective dynamical system by imposing the condition $x^{\prime}=z^{\prime}=0$. We evaluate two critical points for the autonomous Eqs.(17) and (18).

For $P_{1}=(1,1)$, both the eigenvalues are positive showing unstable past attractor. Here $z=1$ implies either $H_{0}=0$ or $H \rightarrow \infty$. Since $H_{0}$ cannot be zero therefore it shows the initial singular cosmic epoch characterized by $H$. We plot the dynamical behavior of critical points corresponding to different values of $\xi$ and $\delta$ as shown in figure 1. In these numerical plots, the white region shows decelerated expansion with $q>0$ while the green region corresponds to $q<0$ showing accelerated expansion of the universe. We observe that both eigenvalues are positive indicating the point $P_{1}$ as an unstable past attractor. This point lies in the physical phase space outside the green region showing decelerated cosmic expansion for all choices of parameters. The point $P_{2}=(1,0)$ corresponds to a saddle point since the eigenvalues have opposite sign. For $P_{3}=\left(1,\left[1+\frac{1}{\xi}\right]^{\frac{1}{\delta-1}}\right)$, the corresponding eigenvalues are negative showing a stable future attractor which lies in green region showing an expanding universe model dominated by viscous matter.

\subsection{Bulk Viscous Matter and Radiation}

We discuss the phase space structure of the model by adding radiation as a new cosmic constituent to study whether the bulk viscous model indicates a radiation dominated epoch. The constraint and Raychaudhuri equations take the form

$$
\begin{gathered}
\rho_{m}+\rho_{r}=3 H^{2}, \\
2 \dot{H}+3 H^{2}=\tilde{\eta} H H_{0}-\frac{1}{3} \rho_{r} .
\end{gathered}
$$

The conservation equation for radiation case can be written as

$$
\dot{\rho}_{r}+4 \rho_{r} H=0 .
$$



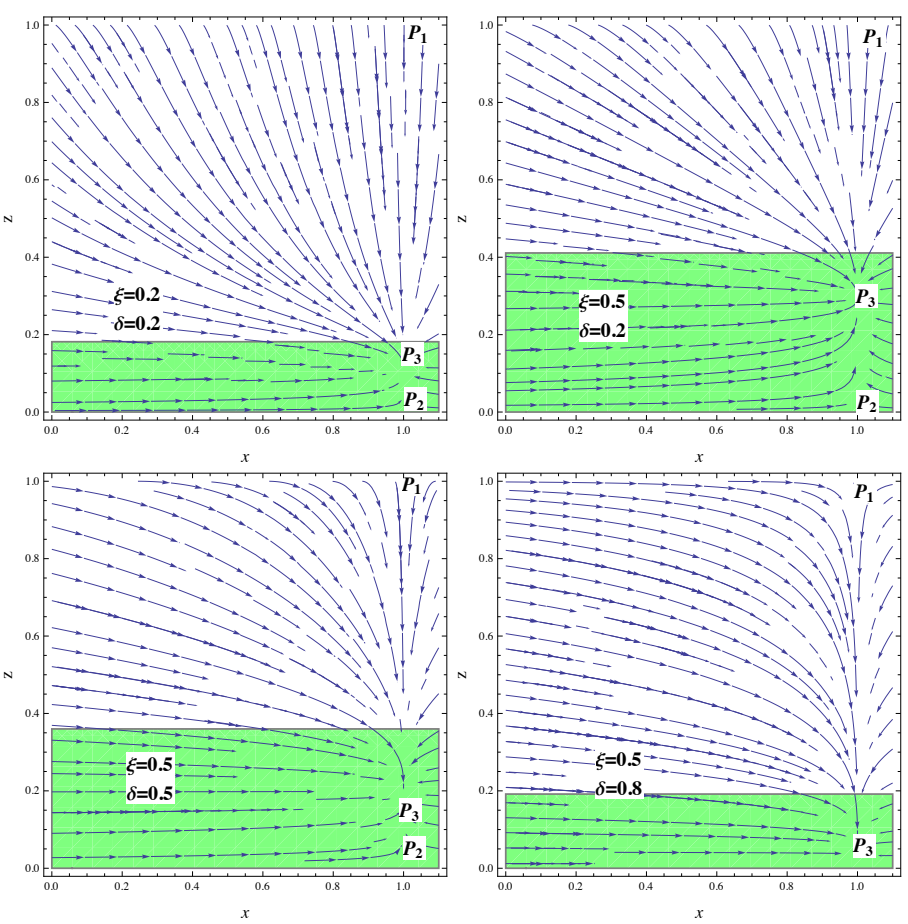

Figure 1. Plots for the phase plane evolution of FRW universe model with different values of $\xi$ and $\delta$.

We generalize the density parameter for radiation by $\Psi_{r}=\frac{\rho_{r}}{3 H^{2}}$ such that Eq.(20) becomes

$$
\dot{H}=\frac{1}{2}\left[\tilde{\eta} H H_{0}-H^{2} \Psi_{r}-3 H^{2}\right] .
$$

The deceleration and EoS parameters are computed as

$$
\begin{gathered}
q=\frac{1}{2}\left[1+\Psi_{r}-3 \xi\left(\frac{H}{H_{0}}\right)^{\delta-1}\right], \\
\omega=\frac{\Psi_{r}}{3}-\xi\left(\frac{H}{H_{0}}\right)^{\delta-1} .
\end{gathered}
$$

We define dimensionless variables as

$$
x=\Psi_{m}=\frac{\rho_{m}}{3 H^{2}}, \quad y=\Psi_{r}=\frac{\rho_{r}}{3 H^{2}}, \quad z=\frac{1}{1+\frac{H_{0}}{H}},
$$

through which, the dynamical system of equations for phase space coordinates become

$$
\begin{aligned}
& x^{\prime}=3 \xi(1-x)\left(\frac{1-z}{z}\right)^{1-\delta}+x y, \\
& y^{\prime}=y\left[y-1-3 \xi\left(\frac{1-z}{z}\right)^{1-\delta}\right],
\end{aligned}
$$


Table 1. Stability Analysis for Matter and Radiation Case

\begin{tabular}{|c|c|c|c|}
\hline Critical Point & Behavior & Stability & Behavior of $q$ \\
\hline$P_{1}$ & Source & Unstable & Deceleration \\
\hline$P_{2}$ & Saddle & Unstable & Deceleration \\
\hline$P_{3}$ & Sink & Stable & Deceleration \\
\hline
\end{tabular}

$$
z^{\prime}=\frac{(1-z)}{2}\left[3 \xi(1-z)\left(\frac{1-z}{z}\right)^{-\delta}-z(y+3)\right] .
$$

We evaluate three critical points by setting $x^{\prime}=y^{\prime}=z^{\prime}=0$. For $P_{1}=\left(x_{c}, y_{c}, z_{c}\right)=(0,1,1)$, the corresponding eigenvalues show an unstable past attractor. This point lies in decelerated expanding phase. The point $P_{2}=(u, 0,1)$ corresponds to a saddle node showing decelerated cosmic expansion.

For $P_{3}=\left(1,0, \frac{1}{1+\frac{1}{\delta-1}}\right)$, the corresponding eigenvalues are negative indicating stable late attractor in deceleration phase for different choices of parameters. The summary of the corresponding results is given in table 1.

\section{Summary}

We have studied phase space analysis for homogeneous and isotropic universe model by taking a mixture of viscous dust and radiating fluid. We have considered a power-law model for the bulk viscous coefficient. Firstly, we have discussed stability of the universe dominated by bulk viscous matter through their eigenvalues corresponding to different values of $\xi$ and $\delta$ (figure 1). We have found an unstable initial matter dominated state undergoing decelerated expansion for $P_{1}$ with all choices of parameters. The point $P_{2}$ is a saddle point in matter dominated universe. The point $P_{3}$ corresponds to a stable matter dominated universe undergoing accelerated expansion. It is observed that the green region (accelerated expansion) tends to increase by increasing $\xi$ while this region gets smaller by increasing $\delta$. It is worth mentioning here that stable solutions (undergoing accelerated expansion) exist in the presence of power-law model of bulk viscosity.

Secondly, we have studied stability of the universe model with bulk viscous radiation and matter to check whether it also indicates a prior radiation dominance. It is found that the critical points $P_{1}$ and $P_{2}$ are saddle/unstable showing initially the matter dominated phase (without acceleration). The point $P_{3}$ is a stable attractor showing decelerated cosmic expansion. We conclude that no stable point (undergoing accelerated expansion) exists with bulk viscous radiation and matter.

\section{References}

[1] Riess, A.G. et al., Astron. J. 116, 1009(1998); Perlmutter, S.J. et al., Astrophys. J. 517, 565(1999); Bennett, C.L. et al., Astrophys. J. Suppl. 148, 1(2003).

[2] Zimdahl, W., Phys. Rev. D 53, 5483(1996).

[3] Guo, Z.K. et al., Phys. Lett. B 608,177(2005).

[4] Yang, R.J. and Gao, X.T., Class. Quantum Grav. 28, 065012(2011).

[5] Sharif, M. and Mumtaz, S., Eur. Phys. J. C 77, 136(2017).

[6] Sharif, M. and Mumtaz, S., arXiv:1706.07659.

[7] Eckart, C., Phys. Rev. 58, 919(1940).

[8] Wang, D. and Meng, X.H., arXiv:1611.01614. 